\title{
Envenomation by stings of reptiles complicated by pulmonary embolism in a child
}

\author{
Ghorbel. S ${ }^{1}$, S. tilouche ${ }^{1}$, R. Kebaiili' ${ }^{1}$ A. Tej ${ }^{1}$, N. Kahloul ${ }^{1}$, J. Bouguila ${ }^{1}$, L. boughamoura ${ }^{1}$. \\ ${ }^{1}$ Farhat Hached Hospital, Pediatric, Sousse, Tunisia.
}

\section{Introduction}

Envenomation by stings of reptiles is a therapeutic emergency. Snake bites are responsible for a high mortality rate in Africa. We report a case of pulmonary embolism, a rare situation in the wake of viperine envenomation in a child. Through this clinical case, we discuss the pathophysiological links between envenomation and disease and the place of heparinotherapy in the management of these patients.

\section{Aims:}

Focus on this particular clinical entity by describing the case collected in our department and by analyzing the data of the literature in order to recall the clinical manifestations, the therapeutic treatment and the evolution of viperin envenomations in children.

\section{Observation :}

* Male, 3 years and 6 months

* Snake bite at the inner edge of the left foot

* He had vomiting and paresthesia of the limb.

* EHD: correct, good neurological and respiratory status

* MIG: edema, redness, pain, Homans + sign

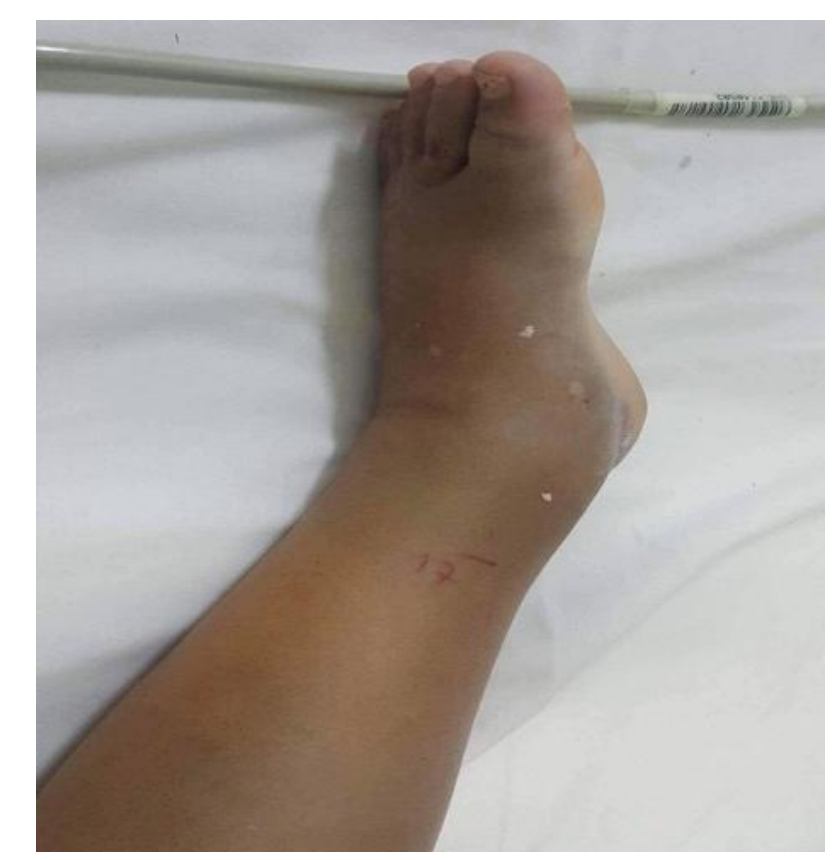

pic 1: appearance of the lower left limb

initial treatment: symptomatic treatment + antivenom immunotherapy and preventive heparin.

Doppler ultrasound: a deep vein thrombosis interesting the femoral vein

So the patient was treated par heparin (curative dose).

\section{Evolution:}

One week after; apparition of respiratory distress .The angio TDM thoraciq showed a massive bilateral pulmonary embolism.
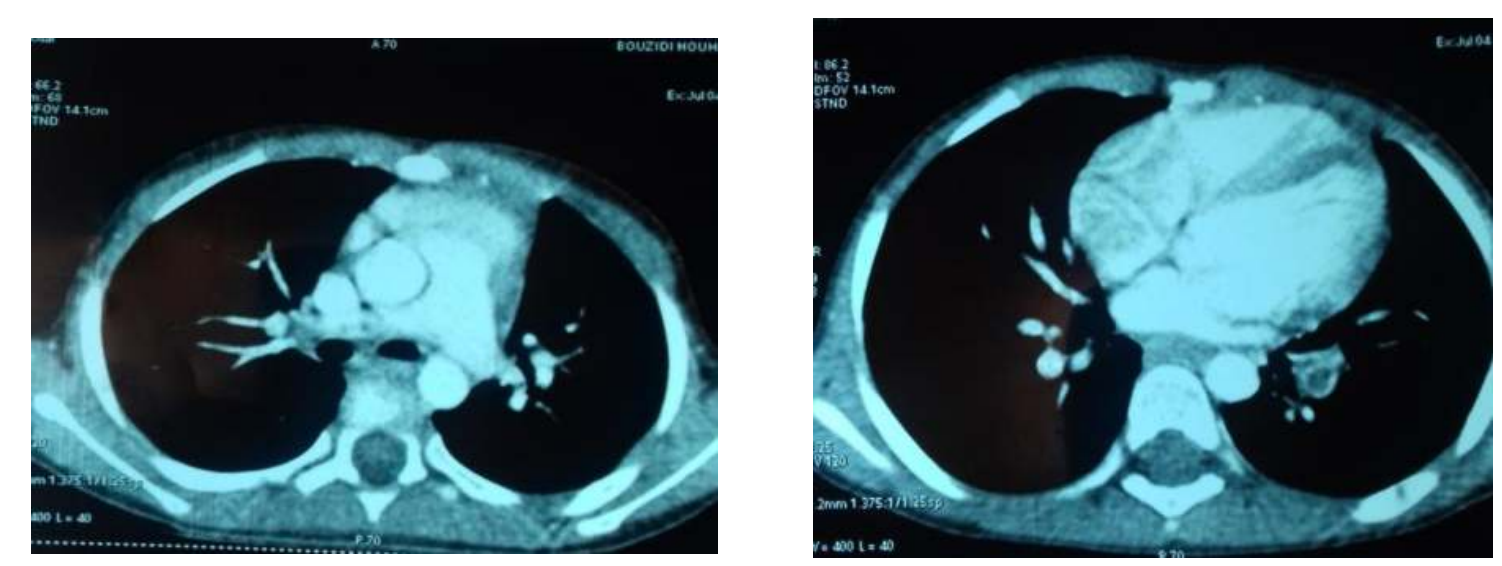

Pic2:thrombosis of the pulmonary artery

* After 36 days, a skin graft was practiced in plastic surgery

* The f evolution was favorable with relay of heparin by anti vitamin $\mathrm{K}$ for six months.

\section{Discussion:}

* Viperian envenomation has been observed in all age groups

* Venous or arterial thromboses are exceptional: not during the acute phase but when an inflammatory syndrome is established.

* Pulmonary embolism is exceptional during viperine envenomation. Only seven cases have been reported in the literature

* In our observation; Pulmonary embolism did not occur until one week after hospitalization: Thrombosis is directly caused by venom, but pulmonary embolism is probably caused by the secondary inflammatory context or by venous thrombus migration.

* The only effective treatment is immunotherapy which is very well tolerated. The initial dose of antivenom (VAS) recommended by slow intravenous infusion is $4 \mathrm{ml}$ : in children, the same dosage should be used as in adults.

* The introduction of heparin as a preventive measure is justified only at a distance from the bite.

\section{Conclusion :}

Pulmonary embolism is an exceptional complication of Viper syndrome. The interest of this observation is to draw attention to the state of hypercoagulability that may appear. 